\title{
Pueblos indigenas y salud colectiva: hacia una ecología de saberes
}

\section{| ${ }^{1}$ Anderson Rocha-Buelvas |}

Resumen: Este ensayo académico pretende articular epistemológicamente el conocimiento indígena y el discurso ético-político de la salud colectiva a través de la propuesta de la ecología de saberes de Boaventura de Souza Santos.

Indudablemente la incursión del conocimiento indígena en las universidades como una nueva agenda académica, requiere que en salud se profundice en el dialogo entre el saber popular y el conocimiento científico. Esta discusión plantea un reto para la salud colectiva desde la justicia cognitiva, la decolonialidad y el conocimiento situado.

> Palabras-clave: población indígena; medicina social; salud pública; justicia social.
1 Universidad Nacional de Colombia, Doctorado en Salud Pública, Bogotá D.C., Colombia (rochabuelvas@gmail.com)

Recebido em:12/05/2017

Aprovado em:08/09/2017 


\section{Introducción}

Posicionadas con más jerarquía en el mundo académico de hoy, las visiones de los pueblos indígenas han dejado de ser estudiadas exclusivamente por el lente de la etnografía y la ciencia eurocéntrica. En su lugar se están generando y discutiendo conocimientos en diversos espacios de la academia a partir de estas mismas visiones (ARÉVALO-ROBLES, 2013). Curiosamente la generación de conocimiento desde y para los pueblos indígenas coincide con los intereses epistemológicos de la salud colectiva, específicamente, respecto a la confrontación con lo que Trouillot (2011) denominaba "racismo epistémico", el cual se ha materalizado en los fundamentos del capitalismo occidental que asume que la realidad es estable y estática porque solo puede verse en una sola perspectiva (MORALES-BORRERO; ROCHA-BUELVAS, 2017), y que la concepción dominante del conocimiento se basa en la evidencia y el método científico que se presenta como una verdad universal sin resistencia y critica. Por consiguiente, resulta provocador que existan iniciativas académicas de origen indígena que estén planteando un paradigma de investigación, ${ }^{1}$ cuya generación y validación de conocimiento (epistemología) no está en sintonía con la ciencia hegemónica. Por el contrario, se acerca a las construcciones ético-políticas y académicas del debate de la salud colectiva. Por tales motivaciones, este manuscrito pretende plantear el reto epistemológico decolonial que tiene la investigación sobre pueblos indígenas desde la salud colectivaque reconoce el proceso de salud-enfermedad, intentando superar el modelo de pensamiento lineal diseñado por el racionalismo europeo y la filosofía positivista. Lo anterior no pretende desconocer la existencia de los aportes que desde el sur ha hecho la importante herramienta conceptual y aplicativa de la epidemiología sociocultural, cuya aproximación dialógica al fenómeno de la enfermedad como proceso colectivo incorpora el proceso saludenfermedad-atención como una expresión relacional que hace uso de saberes y recursos científicos y no científicos (HERSCH-MARTÍNEZ, 2013).

Para este propósito, es necesario comenzar aclarando que el objeto de estudio de la salud colectiva es el proceso salud-enfermedad (PSE) porque asume que la salud no es una categoría atemporal y unívoca, ya que sus prácticas y conocimiento no corresponden a realidades universales, lo que implica que su objeto de estudio no sea estático sino dinámico. Dicho en otras palabras el objeto de estudio de la salud colectiva es un proceso dialectico entre dimensiones de la 
realidad: general, grupal, individual, etcétera, que tienen implicaciones en los campos del saber y de la política (FRANCO, 1993). Lo anterior para afirmar que para superar el racismo cognitivo (CASTRO-GÓMEZ, 2007; QUIJANO, 2007; WALSH, 2009; TROUILLOT, 2011) es necesaria la noción de salud y el conocimiento en salud que corresponden a las premisas del debate de la salud colectiva (MORALES-BORRERO; ROCHA-BUELVAS, 2017).

Una noción de salud que corresponde al proceso vital humano, cuyas características están determinadas por la interdependencia de las múltiples dimensiones presentes en el diario vivir, en las formas de organización social y en las representaciones sobre la propia reproducción social que tienen los sujetos en su contexto histórico, lo cual obliga a avanzar en las ciencias y necesidades de la vida, tanto en sus dimensiones naturales como sociales (FRANCO, 1993); y obliga al reclamo de la justicia cognitiva, ya que una noción de salud amplia comprende la complejidad de los problemas, actores y capitales en salud; y su relación con las formas de injusticia y de una verdadera forma de autodeterminación. El conocimiento en salud por su parte corresponde a la necesidad de transdisciplinariedad y complementariedad, es decir a la necesidad de generar conocimiento a partir de análisis sociales e individuales en un encuadre teórico-metodológico colectivo que se encuentre subordinado a una lógica analítica de procesos dinámicos en una totalidad social. En síntesis, un conocimiento que respete la complejidad de procesos concretos naturales, sociales e históricos (IRIART et al., 2002). Entre tanto, el conocimiento en salud debe corresponder a un producto social condicionado por los procesos históricos y geográficos que guardan relación con el poder. Siendo así construir una noción y un conocimiento de la vida y la salud del mismo proceso vital humano exige la complementariedad de saberes, la transdisciplinariedad y el diálogo intercultural.

En virtud de estas premisas, la generación y socialización del conocimiento en salud colectiva como afirmaba Granda (2004) cuenta con una dimensión material que se refiere a la expresión orgánica, biológica y social (individual y colectiva), y cuenta con una dimensión simbólica encarnada en la interpretación y forma cómo viven y enfrentan estos procesos los sujetos y las colectividades. Lo dicho hasta aquí supone que desde la salud colectiva es posible comprender que las culturas indígenas poseen una lógica diferente que opera dentro de un sistema de sentidopensamiento sobre la naturaleza, es decir, co-razonan con el territorio (FALS 
BORDA, 2009; ESCOBAR, 2014) porque construyen espacio colectivamente a partir de un complejo acervo de creencias-saberes y de prácticas rituales que orientan el corpus del saber indígena (PIÑACUÉACHICUE, 2014). Por todo lo anterior, la generación de conocimiento desde las visiones indígenas en salud colectiva apuntaría a lo que Sousa Santos (2010) ha denominado la ecología de saberes o lo mismo que un dialogo entre el conocimiento científico y el conocimiento popular, una propuesta implícita y generaliza del concepto de interculturalidad en América Latina (REX; SINGH, 2003). Puesto que en la ecología de saberes yace el reto decolonial de la lucha del movimiento indígena y no indígena por reivindicar y legitimar el conocimiento indígena en espacios de interacción entre diversas culturas como los centros de educación superior (PIÑACUÉACHICUE, 2014). Así, la tarea prioritaria de la salud colectiva como campo de pensamiento crítico y de prácticas sociales alternativas será la de mantenerse en construcción y reflexión para superar las injusticias de la matriz de inequidad y explotación del modelo capitalista, cuyas relaciones de opresión están dirigidas al sexo/genero, etnia/raza y clase social, es decir a la subalternidad (BREILH, 2013).

Llama la atención que la intencionalidad de este reto decolonial y su situación epistémica están articulados a los principios ético-políticos y científicos-técnicos de la salud colectiva. Por ejemplo, se requiere una superación dialéctica del enfoque teórico-metodológico para comprender los problemas de cada nación, pueblo y/o comunidad indígena, esto a partir de los conocimientos y las praxis emancipadoras y contrahegemónicas del movimiento indígena, cuya acción social y política sobre los procesos de reproducción social de estas culturas ancestrales reclama una ecología de saberes (ACOSTA; MARTÍNEZ, 2009). Entendiendo que el conocimiento tiene fundamento por medio de la argumentación filosófica sustentada en evidencia lógica, la cual es sistematizada por una ciencia sea occidental o nativa, y que deviene en conocimiento científico teórico o práctico sobre el ser humano, la naturaleza o el cosmos. Entendiendo que la sabiduría se refiere al acontecer, al sentido común, al saber popular, es decir, a una realidad social situada culturalmente, por tanto, es necesario que la salud colectiva con el fin de producir conocimiento desde y para los pueblos indígenas apunte a la ecología de saberes o a las epistemologías del sur, ya que el conocimiento y la sabiduría son procesos de conocimiento con trascendencia histórica (AGÜERO SERVIN, 2011), portanto reclaman justicia cognitiva. En aras de este dialogo 
de saberes, no cabe la menor duda que una noción sobre la cosmovisión sea el punto de partida para la disquisición del conocimiento indígena con el mundo occidental, ya que como lo afirmaba Hart, (2010) la experiencia existencial e histórica de los individuos y colectividades indígenas tienen su propio modo de ver, interpretar y actuar en y sobre el mundo.

Atendiendo a estas consideraciones, la ecología de saberes deberá enfrentar varios retos. En primer lugar reconocer que el pensamiento indígena se instala en los fenómenos de la naturaleza para luego enunciar sus saberes y conocimientos (PIÑACUÉACHICUE, 2014). En segundo lugar, que para los indígenas, la "naturaleza es la única maestra y fuente de sabiduría" (LAME, 2004), es por ello, que la experiencia del territorio-creencia-saber debe ser el principio instituido en el colectivo. En tercer lugar que el conocimiento ancestral se configura en los antepasados no de forma líneal sino a manera de espiral (el pasado es también presente y asimismo es futuro porque los jóvenes retoman el conocimiento de los mayores). Y en cuarto lugar que la práctica del pensamiento indígena se configura a escala "local-global", es decir, las ideas se debaten en un escenario doméstico y se agotan en un escenario multitudinario de discusión colectiva, como lo son los congresos de las organizaciones indígenas (PIÑACUÉACHICUE, 2014).

Entonces la ecología de saberes o el reconocimiento de saberes requiere articular los procesos de producción del conocimiento en cada nación, pueblo y/o comunidad ${ }^{2}$ indígena con la valorización de saberes y conocimientos sobre estos grupos sociales que han sufrido la discriminación y la opresión histórica y sistemática ejercida por la naturalización de las desigualdades orientadas por el capitalismo y el colonialismo (SOUZA SANTOS, 2010).

En esta perspectiva, es prioritario que el reto decolonial por el que venimos trascendiendo, sea abordado desde las premisas de las epistemologías del sur. Éstas son las siguientes.

\section{El mundo es mucho más amplio que la comprensión occidental del mundo}

Esta primera premisa de las epistemologías del sur obliga a reconocer que en las últimas décadas el pensamiento indígena en América Latina como el pensamiento nativo en países angloparlantes surgen como contrapeso a la colonialidad y sus dimensiones, ${ }^{3}$ pues infortunadamente sigue vigente en la ciencia la idea de la 
estratificación socio-racial, donde existen los "blancos" y las otras "tipologías raciales" (GESCO, 2012).

Comprender el mundo más amplio desde las visiones de los pueblos indígenas permite superar la colonialidad del ser y del saber cómo las principales fuentes constitutivas del conocimiento occidental (GESCO, 2012). Para este propósito la salud colectiva debe resistirse a producir una narrativa universal porque descalificaría epistémicamente al otro (LANDER, 2000), incurriría en violencia epistémica y/o racismo epistémico. En ese sentido, resistirse a la comprensión omnipresente de la ciencia occidental por parte de la salud colectiva consistirá en comprender el proceso salud-enfermedad-cuidado-muerte como una construcción individual y social, en tanto, solo puede comprenderse de manera contextualizada en el universo de creencias, valores y comportamientos del medio sociocultural, y de las condiciones materiales de vida de los pueblos indígenas. Principalmente porque comprender el proceso salud-enfermedad-cuidado-muerte desde la ecología de saberes además de explorar prácticas científicas alternativas, deberá promover la interdependencia entre los conocimientos científicos y no científicos, con el objeto de producir experiencias comprensibles del mundo (RUÍZ-LURDUY et al., 2016).

Por consiguiente para conseguir la justicia cognitiva que reclama el diálogo entre conocimiento científico y popular en los pueblos indígenas, es de vital importancia la movilización social y su incipiente incorporación a la academia, pues la experiencia de los movimientos sociales se oponen a la pureza epistémica situada en el punto cero de la observación que desecha otras observaciones y metafísicas mediante la hegemonía de la raza, los saberes modernos, las tecnologías de gobierno y la institucionalización (CASTRO-GÓMEZ, 2004).

\section{La diversidad del mundo es más amplia que la comprensión occidental del mundo}

Esta segunda premisa de las epistemologías del sur plantea que el reto decolonial de la investigación sobre pueblos indígenas desde la salud colectiva es la ecología de saberes producto de la lucha del conocimiento indígena por ser reconocido. La diversidad del mundo implica que la salud colectiva revele que el conocimiento indígena se erige en la ecosofía de la vida o la cosmovisión; por tanto, es un conocimiento que prescinde de las relaciones dualistas de la ciencia hegemónica 
entre mente/cuerpo, pensamiento/acción y sociedad/naturaleza (CAJIGASROTUNDO, 2007). El conocimiento generado desde y para pueblos indígenas como reto decolonial puede ser entonces holístico, cíclico, y relacional porque considera que todo está vivo indiferentemente si es humano o no humano, porque considera que la relación entre las personas y el mundo espiritual es inseparable, y porque los seres humanos importan menos que el mundo (SIMPSON, 2000), es decir, el tiempo, las formas de organizar la vida y la provisión de bienes son concepciones del mundo diversas. De modo que la aceptación de las nociones de alteridad, representación, discurso, identidad, saber-poder en el mundo actual (SIKES, 2006) son una oportunidad para que el conocimiento desde y para pueblos indígenas asuma un proceso de decolonización que se encargue de la recuperación de la cultura, la historia, la identidad, la lengua, la lucha por la ética, la justicia social y la autodeterminación.

El reto decolonial (la ecología de saberes) de la salud colectiva tienen como objetivo lo que denomina Kuokkanen (2000) la consolidación de una "autoestima social”, esto a partir del saber cultural y científico en diálogo. Para ello, la salud colectiva requiere de una postura política en defensa de los derechos colectivos y una posición que repiense su forma de decolonizar.El reto será entonces tomar control sobre las vidas y los territorios indígenas a través de la generación de un nuevo conocimiento que respete sus costumbres, honre sus ritos y reconozca sus cosmovisiones como forma concreta de autodeterminación, existencia y supervivencia (MARTÍN, 2003).

\section{La gran diversidad del mundo no puede ser monopolizada por una teoría general}

En esta tercera premisa es importante asumir que el conocimiento puede ser construido y transformado pluralmente. En favor de la investigación desde y para los pueblos indígenas es necesario que la salud colectiva asuma que la ciencia nativa es inseparable a la cosmovisión indígena, cuyas actuaciones en el mundo son el ritual, la ceremonia y los sueños como puerta a la creatividad y el conocimiento (CAJETE, 2000). La salud colectiva admitirá entonces la gran diversidad del mundo través de la ciencia nativa porque se refiere a los aprendizajes milenarios sistematizados y expresados en la forma de pensar, actuar y percibir del indígena. Por tanto, el reto decolonial desde el punto de vista epistemológico consistirá en 
integrar al conocimiento científico la espiritualidad, el conocimiento humano basado en la cosmología, la perpetuación de los procesos naturales del mundo, el respeto por los lugares-plantas-animales-fenómenos naturales, la interdependencia y el rol de los ancianos como guardianes del conocimiento al proceso vital humano.

Es por lo anterior que dentro de los conceptos de salud y enfermedad de los pueblos indígenas, se puede apreciar la inexistencia de las relaciones dualistas, razón por la cual la salud colectiva tiene un reto epistemológico, puesto que como afirma Nunes (2014) en su construcción ha pretendido asumir un carácter multiparadigmático, que si bien es producto de la convergencia de múltiples epistemologías y campos interdisciplinarios, sigue siendo un encuentro entre paradigmas y conocimientos hegemónicos y eurocéntricos. De modo que es necesario que el conocimiento científico dialogue con la cosmovisiónde los pueblos indígenas como una relación armónica y holística con la naturaleza (SMITH, 1999) que atiende sus problemas de salud bajo la restauración del equilibrio con la naturaleza no curando los síntomas o manifestaciones de la enfermedad como hecho científico externo al individuo (FLORES-GUERRERO, 2004)

\section{Conclusiones}

La ecología de saberes como reto decolonial de la salud colectiva desde y para pueblos indígenas, propende por lo que Wilson (2008) considera la construcción y fortalecimiento de un sistema indígena de investigación como organizador del proceso de conocimiento, es decir, que la ciencia hegemónica debe ser disputada o complementada por un sistema de pensamiento indígena con una propia lógica de pensar, conocer, socializar yresolver. Una lógica que como afirma FernándezLarrinoa (2016) va más allá de lo material porque es un vínculo comunicativo que opera en las esferas de las relaciones de los seres vivos, de los espíritus y de los objetos físicos. Por tanto, el reto decolonial es la diversidad de saberes, cuyos medios de preservación serán los conocimientos anidados al lugar, a la identidad y al principio cósmico. No puede ser de otra forma porque el conocimiento indígena pervive en las leyendas, símbolos, tejidos, danzas, pinturas, ritualesceremonias, y en la lengua. De modo que el conocimiento indígena toma distancia del occidental porque este último es una matriz cognitiva universal, donde el conocimiento para serlo, debe ser considerado válido, legítimo y objetivo (CHILISA, 2012; KOVACH, 2010; WILSON 2008). 
En esta lógica, los pueblos indígenas y la salud colectiva hacia una ecología de saberes debe entender que en las comunidades ancestrales, el mito es el punto de partida para configurar el universo como lo afirma Viveiros de Castro (2002); y debe entender que el modelo de pensamiento de los pueblos indígenas es en espiral no lineal, ya que centran su preocupación en el dialogo y en el vínculo comunicativo, por tanto es incluyente dado que conecta tanto los conocimientos y las experiencias colectivamente como el presente y el pasado, de tal forma que el conocimiento y los procesos históricos en el mundo indígena puede comenzar en cualquier punto del espiral (CAJETE, 2000).

Siendo así, el reto decolonial de la salud colectiva inserto en las epistemologías del sur consiste en revalorizar e implementar el saber ancestral/comunitario como manifestación ético-política de autodeterminación y justicia social mediante la crítica de la relación saber-poder colonial. Por tanto, a todas luces el dialogo de saberes y/o el reclamo por justicia cognitiva serán una interacción racional, espiritual e intuitiva, que en un sentido holístico y complementario, no aísla ni fragmenta la realidad en partes; por el contrario, privilegia las relaciones interdependientes y complementarias de la realidad (ARÉVALO-ROBLES, 2013). Esta ecología de saberes dialoga y argumenta recursivamente con las epistemologías occidentales en aras de que la generación de nuevo conocimiento pueda ser un diagnostico crítico del presente y una reconstrucción, formulación y legitimación de alternativas para una sociedad más justa. Por consiguiente, la lucha de la investigación desde y para pueblos indígenas plantea el diálogo entre mentalidades, sociabilidades, maneras de vivir y convivir.

Para la generación de conocimiento sobre pueblos indígenas desde la salud colectiva se requiere asumir como lo propone Almeida-Filho (2006) las propiedades de los objetos de conocimiento en dialogo como eje para unificar parcialmente las variadas contribuciones, a propósito de este manuscrito, las que apuntan a un reto decolonial que busca desmantelar la lógica reduccionista del positivismo que simplifica la realidad en busca de su esencialidad y que irrespeta la complejidad inherente a los procesos concretos de la naturaleza, sociedad e historia. Por ello, los sustantivos comunes de la teoría crítica europea que determinan el debate de la salud colectiva como también las epistemologías del sur, están siendo representados hoy por los grupos sociales invisibles o subalternos. Una invisibilidad reparable que ha sido originada y reproducida por la ausencia de un diálogo entre las 
ideas y las creencias, es decir, entre la ciencia y la subalternidad o el movimiento social, ejemplo de ello la ausencia de diálogo entre universidades y movimientos sociales o la criminalización de ese diálogo cuando surge. Esta realidad puede ser transformada por la contribución de la salud colectiva y otros campos de pensamiento crítico que pretenden realizar investigación desde y para pueblos indígenas. La salud colectiva como heredera de la medicina social debe ejercer su epistemología como ciencia crítica y con las luchas de las organizaciones sociales en contra de la dominación a partir del profundo cuestionamiento epistemológico y ético-político que producen conocimiento.

\section{Referencias}

ACOSTA, A.; MARTÍNEZ, E. El buen vivir. Una vía para el desarrollo. Quito, Ecuador: AbyaYala, 2009.

ALMEIDA-FILHO, N. Complejidad y transdisciplinariedad en el campo de la Salud Colectiva: evaluación de conceptos y aplicaciones. Salud colectiva, v. 2, n. 2, p. 123-146, 2006. ARÉVALO-ROBLES, G.A. Reportando desde un frente decolonial: a la emergencia del paradigma indígena de investigación. In: ARÉVALO-ROBLES, G.A.; CHAUSTRE, I. Z. (Coord.) Luchas, experiencias y resistencia en la diversidad y la multiplicidad. Bogotá, Colombia: MunduBerriak, 2013 (Cuadernos de Trabajo, 2).

BREILH, J. La determinación social de la salud como herramienta de transformación hacia una nueva salud pública (salud colectiva). Revista Facultad Nacional de Salud Pública, v. 31, supl. 1, p. 13-27, 2013.

CAJETE, G. Native science: natural laws of interdependence. Santa Fe, NM, USA: ClearLight Publishing, 2000.

CAJIGAS-ROTUNDO, J.C. La biocolonialidad del poder. Amazonía, biodiversidad y ecocapitalismo. In: CASTRO-GÓMEZ, S.; GROSFOGUEL, R. El giro decolonial: reflexiones para una diversidad epistémica más allá del capitalismo global. Bogotá: Siglo del Hombre Editores, 2007.

CASTRO-GÓMEZ, S. La hybris del punto cero. Ciencia, raza e ilustración en la nueva granada (1750-1816). Bogotá: Pontificia Universidad Javeriana/Instituto Pensar, 2004.

CASTRO-GÓMEZ, S.; GROSFOGUEL, R. Elgiro decolonial: reflexiones para una diversidad epistémica más allá del capitalismo global. Bogotá: Siglo del Hombre Editores, 2007.

CHILISA, B. Indigenous research methodologies. London: Sage, 2012.

AGÜERO SERVIN, M.M. Conceptualización de los saberes y el conocimiento. Revista Decisio, n. 30, p. 16-20, 2011. 
SOUZA SANTOS, B. El milenio huérfano. Ensayos para una nueva cultura política. Madrid y Bogotá: Editorial Trotta S, 2005.

SOUSA SANTOS, B. Para descolonizar Occidente: más allá del pensamiento abismal. Buenos Aires: Prometeo Libros, 2010.

ESCOBAR, A. Sentipensar con la tierra. Nuevas lecturas sobre desarrollo, territorio y diferencia. Medellín: Ediciones UNAULA, 2014 (Colección Pensamiento vivo).

FALS BORDA, O. Una sociología sentipensante para América Latina. Bogotá: Siglo del Hombre Editores y CLACSO, 2009.

FERNÁNDEZ-LARRINOA, K. Paradigma de investigación y pueblos indígenas del primer mundo: crítica cultural del trabajo social. Revista Andaluza de Antropología, n. 10, p. 54-78, 2016.

FLORES-GUERRERO, R. Salud, enfermedad y muerte: lecturas desde la antropología sociocultural. Revista Mad, v. 0, n. 10, p. 21-29, 2004.

FRANCO, S. Proceso vital humano, proceso saludlenfermedad: una nueva perspectiva. In: SEMINARIO MARCOCONCEPTUALPARALAFORMACIÓN DEPROFESIONALES DE LA SALUD. Universidad Nacional de Colombia, Bogotá, pp. 1-8, 1993.

GRANDA, E.A qué llamamos salud colectiva. Hoy. Revista Cubana de Salud Pública, v. 30, n. 2, 2004.

GRUPO DE ESTUDIOS SOBRE COLONIALIDAD (GESCO). Estudios Decoloniales: Un panorama general. KULA: Antropólogos Del Atlántico Sur, p. 8-21, 2012.

HART, M.A. Indigenous worldviews, knowledges, and research: the development of an indigenous research paradigm. Journal of Indigenous Voices in Social Work, n. 1, p. 1-16, 2010. HERSCH-MARTÍNEZ, P. Epidemiología sociocultural: una perspectiva necesaria. Salud Pública de México, v. 55, p. 512-518, 2013.

IRIART, C. et al. Medicina social latinoamericana: aportes y desafíos. Revista Panamericana de Salud Pública, n. 12, p. 128-136, 2002.

KOVACH, M. Indigenous methodologies: characteristics, conversations, and contexts. University of Toronto Press, Toronto, 2010.

KUOKKANEN, R. Towards an indigenous paradigm from a Sami perspective. The Canadian Journal of NativeStudies, v. 20, n. 2, p. 411-436, 2000.

LANDER, E. La colonialidad del saber: eurocentrismo y ciencias sociales. Perspectivas latinoamericanas. Buenos Aires: CLACSO, 2000.

LAME, M.Q. Los pensamientos del indio que se educó dentro de las selvas colombianas. Popayán: Universidad del Cauca, 2004.

NUNES, E.D. Paradigmas de la salud colectiva: breve reflexión. Salud colectiva, v. 10, n. 1, p. 57-65, 2014. 
MARTÍN, K. L. Ways of knowing, ways of being and ways of doing: a theoretical framework and methods for indigenous re-search and indigenist research. Voicing Dissent, New Talents 21C: Next Generation. Australian Studies Journal of Australian Studies, v. 76, p. 203-214, 2003.

MORALES-BORRERO, M.C.; ROCHA-BUELVAS, A. Debate entre salud colectiva y salud pública. In: BORRERO, M. C. M. (Ed.). Salud colectiva y salud pública ¿Se está hablando de lo mismo? Bogotá: Universidad Nacional de Colombia, 2017. p.31-44.

PIÑACUÉ ACHICUE, J.C. Pensamiento indígena, tensiones y academia. Tabula Rasa, n. 20, p. 161-192, 2014.

PORSANGER, J.An essayaboutindigenous methodology. Nordlit. Arbeidstidsskriftilitteratur, v. 8, n. 1, p. 105-119, 2004.

QUIJANO, A. Colonialidad del poder y clasificación social. In: CASTRO-GÓMEZ, S.; GROSFOGUEL, R. El giro decolonial: reflexiones para una diversidad epistémica más allá del capitalismo global. Bogotá: Siglo del Hombre Editores, 2007. p. 93-126.

REX, J.; SINGH, G. Pluralism and multiculturalism in colonial and post-colonialsociety - thematic introduction. In: KOENIG, M. (Ed.). Pluralism and multiculturalism in colonial and post-colonial societies. París: Unesco, 2003. p. 106-118.

RUÍZ-LURDUY, R. et al. Desarrollo social y salud pública. Reflexiones en torno a la interculturalidad. Revista Facultad Nacional de Salud Pública, Septiembre-Diciembre, p. 380-388, 2016.

SIKES, P. Decolonizing research and methodologies: indigenous peoples and cross-cultural contexts. Pedagogy, Culture and Society, v. 14, n. 3, p. 349-358, 2006.

SMITH, L. Decolonizing methodologies: research and indigenous peoples. London: ZedBooks, 1999.

SIMPSON, L. B. Anishinaabe ways of knowing. In: OAKES, J. et al. (Eds.). Aboriginal health, identity and resources. Winnipeg, Manitoba: Native Studies Press, 2000. p. 165-185.

TROUILLOT, M.R. Transformaciones globales, la antropología y el mundo moderno. Popayán: Universidad del Cauca, 2011.

VIVEIROS DE CASTRO, E. Perspectivismo e multinaturalismo na América indígena. In: . (Ed.). A inconstância da alma selvagem (e otros ensaios de antropologia). São Paulo: Cosac \& Naify, 2002. p. 345-400.

WALSH, C. Interculturalidad, plurinacionalidad y razón decolonial: refundares políticoepistémicos en marcha. In: ALBAGLI, S.; MACIEL, L. M. (Eds.). Conocimiento, capital y desarrollo: dialécticas contemporáneas.Buenos Aires: Editora La Crujía, 2009.

WILSON, S. Research is ceremony: indigenous research methods. Nova Scotia: Fernwood Publishing, 2008. 
${ }^{1}$ Cf. Smith (1999); Cajete (2000); Kuokkanen (2000); Wilson (2008); Martín (2003); Porsanger (2004); Chilisa (2012); y Kovach (2010).

${ }^{2}$ Se evita utilizar el término grupo étnico, ya que la noción de etnicidad fue un desarrollo del proceso de expansión colonialista europea.

${ }^{3}$ La triple dimensión está constituida por el racismo, el eurocentrismo epistémico y la occidentalización violenta de los estilos de vida. 


\section{Abstract}

\section{Indigenous peoples and Collective Health: towards an ecology of knowledge}

This academic essay aims to articulate epistemologically indigenous knowledge and the ethical-political discourse of collective health through the proposal of the ecology of knowledges of Boaventura de Souza Santos. Undoubtedly, the incursion of indigenous knowledge in universities as a new academic agenda requires that the dialogue between popular knowledge and scientific knowledge be deepened in the field of health. This discussion proposes a challenge for collective health from cognitive justice, decoloniality and situated knowledge.

> Keywords: Indigenous population; social medicine; public health; social justice. 
Povos indigenas e Saúde Coletiva: para uma ecologia do conhecimento

Este ensaio acadêmico procura articular epistemologicamente o conhecimento indígena $\mathrm{e}$ o discurso ético-político da saúde coletiva através da proposta da ecologia dos saberes de Boaventura de Souza Santos. Indubitavelmente, a incursão do conhecimento indígena nas universidades como uma nova agenda acadêmica exige que, na saúde, o diálogo entre conhecimento popular e conhecimento científico seja aprofundado. Esta discussão representa um desafio à saúde coletiva da justiça cognitiva, da descolonialidade e do conhecimento situado.

> Palavras-chave: população indígena; medicina social; Saúde Pública, justiça social. 